\begin{tabular}{|c|l|}
\hline Title & Mean-field effects on collective flow in high-energy heavy-ion collisions at 2-158A GeV energies \\
\hline Author(s) & Isse, M.; Ohnishi, A.; Otuka, N.; Sahu, P. K.; Nara, Y. \\
\hline Citation & $\begin{array}{l}\text { Physical Review C, 72(064908), 1-15 } \\
\text { https://doi.org/10.1103/PhysRevC.72.064908 }\end{array}$ \\
\hline Issue Date & 2005-12-29 \\
\hline Doc URL & http://hdl.handle.net/2115/1401 \\
\hline Rights & Copyright $\odot 2005$ A merican Physical Society \\
\hline Type & article \\
\hline File Information & PRC72-6.pdf \\
\hline
\end{tabular}

Instructions for use 


\title{
Mean-field effects on collective flow in high-energy heavy-ion collisions at 2-158A GeV energies
}

\author{
M. Isse* and A. Ohnishi \\ Division of Physics, Graduate School of Science, Hokkaido University, Sapporo, Hokkaido 060-0810, Japan
}

N. Otuka

Nuclear Data Center, Department of Nuclear Energy System, Japan Atomic Energy Research Institute, Tokai, Ibaraki 319-1195, Japan

P. K. Sahu
Institute of Physics, Sachivalaya Marg, Bhubaneswar 751 005, India

Y. Nara

Institut für Theoretische Physik, Johann Wolfgang Goethe-Universität, Max-von-Laue-Straße 1, D-60438 Frankfurt am Main, Germany

(Received 13 March 2005; published 29 December 2005)

\begin{abstract}
Collective flows in heavy-ion collisions from AGS $[(2-11) A \mathrm{GeV}]$ to SPS $[(40,158) A \mathrm{GeV}]$ energies are investigated in a nonequilibrium transport model with the nuclear mean field (MF). Sideward $\left\langle p_{x}\right\rangle$, directed $v_{1}$, and elliptic flows $v_{2}$ are systematically studied with different assumptions for the nuclear equation of state (EOS). We find that the momentum dependence on the nuclear MF is important for the understanding of the proton collective flows at AGS and SPS energies. Calculated results with momentum-dependent MF qualitatively reproduce the experimental data of proton sideward, directed, and elliptic flows in a incident energy range of (2-158)A GeV.
\end{abstract}

DOI: 10.1103/PhysRevC.72.064908

PACS number(s): 25.75.Ld, 24.10.-i

\section{INTRODUCTION}

Determining the nuclear equation of state (EOS) under various conditions has been one of the principle goals of heavy-ion physics in these decades [1-34]. Near the saturation density, the EOS gives the bulk properties of nuclei such as the binding energy and the radius. While the first-principle simulations of lattice QCD are possible for hot baryon-free nuclear matter, and while matter at low baryon densities can be studied by expanding in the power series of the baryon chemical potential $\mu$ [35], the properties of highly compressed matter are still under debate. Thus phenomenological studies are necessary to connect the experimental heavy-ion collision data with the EOS, especially for nuclear matter at high baryon densities. In high-energy heavy-ion collisions, where nuclear matter over a wide range of temperatures and densities is probed, many ideas on the EOS and phases have been examined. For example, very dense matter was created in recent RHIC experiments [36], suggesting the creation of a gas of deconfined quarks and gluons (QGP). In the 1970's and 1980's the existence of strong collective flow in heavy-ion collisions was suggested in hydrodynamics [1-3], and it was examined in experiments at Bevalac [4]. Since collective sideward flows are generated in the early stages of collisions by the repulsive nucleon potential in nuclear matter, the observed strong collective flows were believed to signal very large pressure at high baryon densities, i.e., the hard EOS [5]. On the other hand, the real part of the nucleon-nucleus potential is already repulsive at normal density at high incident energies,

*Electronic address: isse@nucl.sci.hokudai.ac.jp and the role of the momentum dependence of the nuclear potential in the collective flows was extensively studied after 1990 [5-9]. To distinguish the momentum and the density dependences, we need to invoke heavy-ion collision data over a wide incident energy range. We now have systematic collective flow data at various incident energies from the LBNL Bevalac [10-12], GSI Schwerionen Synchrotron (SIS) [13-15], MSU NSCL [16], BNL Alternating Gradient Synchrotron (AGS) [17-21], CERN Super Proton Synchrotron (SPS) [22-24], and BNL RHIC [37].

Collective flow data obtained at AGS energies [(2-11)A $\mathrm{GeV}$ ] provide a good landmark to determine the EOS. As demonstrated in Ref. [25], the saturating momentum dependence of the mean-field (MF), a large number of hadronic resonances, and string degrees of freedom are essential in order to explain all of the radial, sideward, and elliptic flows at AGS energies. The momentum dependence of nuclear potentials in the context of collective flow was also discussed in Ref. [9] with a quantum molecular dynamics (QMD) model. It is suggested that we can separate the momentum dependence by analyzing the so-called balance energy at which the flow disappears, and this was later confirmed by experiment at NSCL [16] for $E_{\mathrm{inc}}=(55-155) A \mathrm{MeV}$. Recently, Danielewicz and others discussed the EOS with these data within a Boltzmann equation simulation [17,26-28], showing that a reliable stiffness value $(K=167-380 \mathrm{MeV})$ cannot be uniquely determined from currently available collective flow data $\left(F\right.$ or $\left.v_{2}\right)$ up to AGS energies $\left[E_{\text {inc }}=(0.15-\right.$ 11) $A \mathrm{GeV}$ ] [28]. On the other hand, a description comparable with theirs was also obtained in the relativistic BoltzmannUehling-Uhlenbeck (RBUU) model [25] by using a relativistic mean field. In RBUU, the MF is fitted to reproduce the real part of the global optical potential in Dirac phenomenology [38]. In 
Ref. [25] a common MF giving $K \sim 300 \mathrm{MeV}$ is applied in the energy range of $(0.25-11) A \mathrm{GeV}$. Thus these two studies do not necessarily provide the same conclusion for the stiffness. In addition, we still have large ambiguities in the MF for hadrons other than nucleons. In order to reduce these ambiguities and to pin down the EOS more precisely, recently measured flow data at lower SPS energies [(20-80)A GeV] may be helpful, because a higher baryon density would be reached at these incident energies.

Several hadronic transport models, such as RQMD [39-43], BEM [26-28], RBUU [25,32,33], ARC [44], ART [45], HSD [46], UrQMD [47,48], and JAM [49], have been successfully applied to describe many aspects of high-energy heavy-ion collisions over a wide range of incident energies. Transport models without MF effects (ARC, HSD, JAM) can describe bulk observables such as transverse mass spectra or rapidity distributions, but they cannot explain anisotropic collective flows, which are sensitive to MF potentials. Transport models with MF effects (RQMD, BEM, RBUU, ART, UrQMD) have been successful in explaining anisotropic collective flows in addition to bulk observables up to AGS energies. For SPS energies, however, the MF effects on collective flows have not been seriously investigated.

In this work, we investigate collective flows from $2 A \mathrm{GeV}$ to $158 A \mathrm{GeV}$ by using a hadronic cascade model, the jet AA microscopic transportation model (JAM) [49], combined with a covariant prescription of the MF (RQMD/s) [43].

This paper is organized as follows. In Sec. II we explain our transport model and the parametrization of our EOS used there. In Sec. III we present our results for flows on rapidity and transverse distributions as well as their excitation functions. In Sec. IV we discuss some uncertainties in our model. In Sec. V we summarize our work.

\section{NONEQUILIBRIUM TRANSPORT MODEL AND THE EQUATIONS OF STATE}

Heavy-ion collision is a dynamical process of a system in which the temperature and density are not uniform and the equilibrium is not necessarily reached. Therefore we need dynamical models to describe collisions in order to extract static properties of nuclear matter under equilibrium. A hydrodynamic description is the most direct way to connect the EOS and dynamics. Actually, ideal hydrodynamics has succeeded in describing elliptic flow at low $p_{T}$, up to semicentral and around mid-rapidity at RHIC [50] (however, see Ref. [51] for a recent reinterpretation of the RHIC data), where the number of produced particles is so large that local equilibrium may be easily achieved. However the condition of local equilibrium may not be satisfied up to SPS energies, and nonequilibrium dynamics is required for studying the EOS of dense nuclear matter through heavy-ion collisions.

Hadron-string cascade processes are the main source of thermalization and particle production up to SPS energies. In the increase of incident energy from AGS [(2-11)A GeV] to SPS [(20-158)A GeV], the main particle production mechanism in hadron-hadron collisions evolves from resonance productions to string formations. At higher energies, hard partonic interaction (jet production) becomes more important, and the jet production cross section reaches around $20 \%$ of the total cross section of $p p$ at RHIC [52].

JAM includes all of the above particle and jet production mechanisms, and the applicable incident energy range is expected to be adequate (for the study of collective flow, jet production does not matter). Inelastic hadron-hadron collisions produce resonances at low energies. We explicitly include all established hadronic states with masses up to around $2 \mathrm{GeV}$ with explicit isospin states as well as their antiparticles, which are made to propagate in space-time. At higher energies ( $\sqrt{s} \gtrsim 4 \mathrm{GeV}$ in $B B$ collisions, $\sqrt{s} \gtrsim 3 \mathrm{GeV}$ in $M B$ collisions, and $\sqrt{s} \gtrsim 2 \mathrm{GeV}$ in $M M$ collisions) color strings are formed, and they decay into hadrons after their formation time $(\tau \sim$ $1 \mathrm{fm} / \mathrm{c}$ ) according to the Lund string model PYTHIA [53]. Leading hadrons having constituent quarks can scatter within their formation time with other hadrons, assuming the additive quark cross section that is known to be important at SPS energies [48].

It is necessary to include MF effects to explain collective flow data, and the MF should have momentum dependence as well as density dependence in order to describe flows over a wide incident energy range. We adopt here a simple Skyrmetype density-dependent MF in the zero-range approximation and a Lorentzian-type momentum-dependent MF [6] that simulates the exchange term (Fock term) of the Yukawa potential. The single-particle potential $U$ then has the form

$$
\begin{aligned}
U(\boldsymbol{r}, \boldsymbol{p})= & \alpha\left(\frac{\rho(\boldsymbol{r})}{\rho_{0}}\right)+\beta\left(\frac{\rho(\boldsymbol{r})}{\rho_{0}}\right)^{\gamma} \\
& +\sum_{k=1,2} \frac{C_{\mathrm{ex}}^{(k)}}{\rho_{0}} \int d \boldsymbol{p}^{\prime} \frac{f\left(\boldsymbol{r}, \boldsymbol{p}^{\prime}\right)}{1+\left[\left(\boldsymbol{p}-\boldsymbol{p}^{\prime}\right) / \mu_{k}\right]^{2}} .
\end{aligned}
$$

This MF potential leads to the following total potential energy, through the relation $U=\delta V / \delta f$ :

$$
\begin{aligned}
V= & \int d \boldsymbol{r}\left[\frac{\alpha \rho^{2}(\boldsymbol{r})}{2 \rho_{0}}+\frac{\beta \rho^{\gamma+1}(\boldsymbol{r})}{(1+\gamma) \rho_{0}^{\gamma}}\right] \\
& +\sum_{k=1,2} \frac{C_{\mathrm{ex}}^{(k)}}{2 \rho_{0}} \int d \boldsymbol{r} d \boldsymbol{p} d \boldsymbol{p}^{\prime} \frac{f(\boldsymbol{r}, \boldsymbol{p}) f\left(\boldsymbol{r}, \boldsymbol{p}^{\prime}\right)}{1+\left[\left(\boldsymbol{p}-\boldsymbol{p}^{\prime}\right) / \mu_{k}\right]^{2}}
\end{aligned}
$$

where $f(\boldsymbol{r}, \boldsymbol{p})$ is the phase space distribution function whose integral over $\boldsymbol{p}$ is normalized to the density $\rho(\boldsymbol{r})$. At zero temperature the phase space distribution function is given as

$$
f(\boldsymbol{r}, \boldsymbol{p})=\left(\frac{4}{3} \pi p_{F}^{3}\right)^{-1} \rho(\boldsymbol{r}) \Theta\left(p_{F}-|\boldsymbol{p}|\right) .
$$

Then the total energy per nucleon is

$$
\begin{aligned}
\frac{E}{A}(\rho)= & \frac{3}{5} \frac{p_{F}(\rho)^{2}}{2 m}+\frac{\alpha}{2 \rho_{0}} \rho+\frac{\beta}{(1+\gamma) \rho_{0}^{\gamma}} \rho^{\gamma}+\frac{\rho}{2 \rho_{0}}\left(\frac{4}{3} \pi p_{F}^{3}\right)^{-2} \\
& \times \int_{0}^{p_{F}} d \boldsymbol{p} \int_{0}^{p_{F}} d \boldsymbol{p}^{\prime} \sum_{k=1,2} \frac{C_{\mathrm{ex}}^{(k)}}{1+\left[\left(\boldsymbol{p}-\boldsymbol{p}^{\prime}\right) / \mu_{k}\right]^{2}}
\end{aligned}
$$


TABLE I. Parameter set of density-dependent and momentum-dependent/independent potentials. Momentum-dependent hard (MH) and soft (MS) potentials are taken from Ref. [55] with simplification (see text for detail). Momentum-independent hard (H) and soft (S) potentials are taken from Ref. [56].

\begin{tabular}{lcccccccc}
\hline \hline Type & $\begin{array}{c}\alpha \\
(\mathrm{MeV})\end{array}$ & $\begin{array}{c}\beta \\
(\mathrm{MeV})\end{array}$ & $\gamma$ & $\begin{array}{c}C_{\mathrm{ex}}^{(1)} \\
(\mathrm{MeV})\end{array}$ & $\begin{array}{c}C_{\mathrm{ex}}^{(2)} \\
(\mathrm{MeV})\end{array}$ & $\begin{array}{c}\mu_{1} \\
\left(\mathrm{fm}^{-1}\right)\end{array}$ & $\begin{array}{c}\mu_{2} \\
\left(\mathrm{fm}^{-1}\right)\end{array}$ & $\begin{array}{c}K \\
(\mathrm{MeV})\end{array}$ \\
\hline MH & -33 & 110 & $5 / 3$ & -277 & 663 & 2.35 & 0.4 & 448 \\
MS & -268 & 345 & $7 / 6$ & -277 & 663 & 2.35 & 0.4 & 314 \\
H & -124 & 70.5 & 2 & - & - & - & - & 380 \\
S & -356 & 303 & $7 / 6$ & - & - & - & - & 200 \\
\hline \hline
\end{tabular}

where the Fermi momentum is taken to be $p_{F}(\rho)=$ $\hbar\left(3 \pi^{2} \rho / 2\right)^{1 / 3}$. See Eq. (A22) for the definition of $\rho$ used in the actual simulations. Integrals in Eq. (4) can be obtained analytically [7] as

$$
\begin{array}{rl}
\int_{0}^{p_{F}} & d \boldsymbol{p} \int_{0}^{p_{F}} d \boldsymbol{p}^{\prime} \frac{1}{1+\left[\left(\boldsymbol{p}-\boldsymbol{p}^{\prime}\right) / \mu\right]^{2}} \\
= & \frac{32 \pi^{2}}{3} p_{F}^{4} \mu^{2}\left\{\frac{3}{8}-\frac{\mu}{2 p_{F}} \arctan \frac{2 p_{F}}{\mu}-\frac{\mu^{2}}{16 p_{F}^{2}}\right. \\
+ & \left.\left[\frac{3}{16} \frac{\mu^{2}}{p_{F}^{2}}+\frac{1}{64} \frac{\mu^{4}}{p_{F}^{4}} \ln \left(1+\frac{4 p_{F}^{2}}{\mu^{2}}\right)\right]\right\} .
\end{array}
$$

Parameters $\alpha, \beta$, and $\gamma$ in Eq. (4) are determined to reproduce the saturation of the total energy per nucleon at the normal nuclear density, i.e., $E /\left.A\right|_{\rho=\rho_{0}}=-16 \mathrm{MeV}$ and $P=$ $\rho^{2} \partial(E / A) /\left.\partial \rho\right|_{\rho=\rho_{0}}=0 \mathrm{MeV} / \mathrm{fm}^{3}$ [54]. The incompressibility $K$ is obtained from $K=9 \rho^{2} \partial^{2}(E / A) /\left.\partial \rho^{2}\right|_{\rho=\rho_{0}}$. Parameters for the hard $(\mathrm{H})$ and soft $(\mathrm{S})$ EOS are listed in Table I, and the density dependences of the total energy per nucleon are shown in the right-hand panel of Fig. 1.

Parameters $C_{\mathrm{ex}}^{(k)}$ and $\mu_{k}$ are taken to reproduce the real part of the global Dirac optical potential (Schrödinger equivalent potential) of Hama et al. [38], in which angular distribution and polarization quantities in proton-nucleus elastic scatterings are analyzed in the range of $10 \mathrm{MeV}$ to $1 \mathrm{GeV}$ in Dirac phenomenology. The single-particle potential at $\rho=\rho_{0}$,

$$
\begin{aligned}
U\left(\boldsymbol{p}, \rho_{0}\right)= & \alpha+\beta+\left(\frac{4}{3} \pi p_{F}^{3}\right)^{-1} \\
& \times \int_{0}^{p_{F}} d \boldsymbol{p}^{\prime} \sum_{k=1,2} \frac{C_{\mathrm{ex}}^{(k)}}{1+\left[\left(\boldsymbol{p}-\boldsymbol{p}^{\prime}\right) / \mu_{k}\right]^{2}} \\
= & \alpha+\beta+\left(\frac{4}{3} \pi p_{F}^{3}\right)^{-1} \\
& \times \sum_{k=1,2} C_{\mathrm{ex}}^{(k)} \pi \mu_{k}^{3}\left[\frac{p_{F}^{2}+\mu_{k}^{2}-p^{2}}{2 p \mu_{k}}\right. \\
& \ln \frac{\left(p+p_{F}\right)^{2}+\mu_{k}}{\left(p-p_{F}\right)^{2}+\mu_{k}}+\frac{2 p_{F}}{\mu_{k}} \\
& \left.-2\left(\arctan \frac{p+p_{F}}{\mu_{k}}-\arctan \frac{p-p_{F}}{\mu_{k}}\right)\right],
\end{aligned}
$$

is compared with the Schrödinger equivalent potential from Ref. [38] in the left-hand panel of Fig. 1. Parameters for the momentum-dependent potentials are shown as $\mathrm{MH}$ and MS in Table I. These parameter sets are based on Ref. [55] with a simplification in which the Coulomb, surface, and Pauli potentials as well as the zero-point kinetic energy of the Gaussian wave packets are dropped, because their study focused on nuclear matter below the saturation density. We have fixed the high-energy limit of the optical potential $U \rightarrow 77 \mathrm{MeV}$ at $E_{\mathrm{inc}} \rightarrow \infty$, leading to a constraint $\alpha+\beta=$ $77 \mathrm{MeV}$. This constraint generally makes the EOS stiffer than those in Ref. [31].
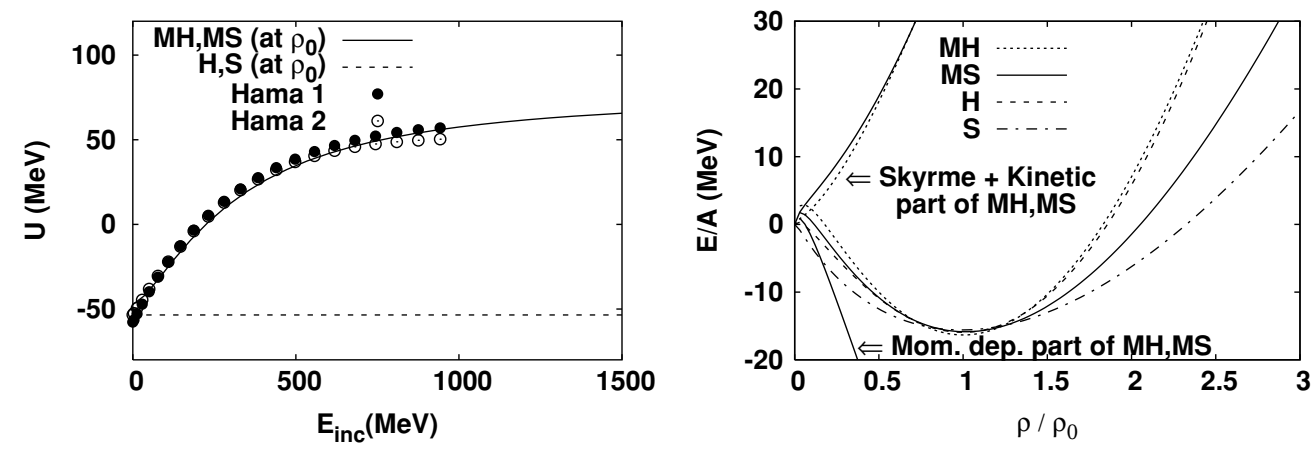

FIG. 1. Left, momentum dependence of the single-particle potentials, Eq. (6), for momentum-dependent hard (MH) and soft (MS) and momentum-independent hard $(\mathrm{H})$ and soft $(\mathrm{S})$ potentials are compared with the real part of the global Dirac optical potential [38]. Right, density dependence of total energy per nucleon in Eq. (4) for momentum-dependent (MH, MS) and -independent (H, S) potentials. 
We include the above MF effects in JAM [49] by means of simplified RQMD (RQMD/S) [43] framework. The relativistic quantum molecular dynamics (RQMD) model $[39,40,42]$ is a constrained Hamiltonian dynamics, in which potentials are treated in a covariant way. RQMD/S [43] uses much simpler and more practical time fixation constraints than the original RQMD $[39,40,42]$. For details, see Appendix.

In this work we take into account potential interactions only between baryons. The simulation time step size is taken to be $d t=0.1 \mathrm{fm} / c$ at all incident energies. We discuss the influence of MF for nonnucleonic baryons on the flow analysis and the validity of this treatment in Sec. IV. The magnitude of the energy conservation violated is about $0.4 \%$ on average for time and events.

\section{COLLECTIVE FLOWS FROM AGS TO SPS ENERGIES}

When two heavy nuclei collide at high energies at finite impact parameters, the pressure gradient is anisotropic in the initial stages of the collision. As a result, it generates anisotropic collective flows. Until now, several kinds of collective flows have been proposed for probing highly dense matter. The first is the sideward flow (also called directed flow) $\left\langle p_{x}\right\rangle$, which is defined as the mean value of $p_{x}$, where $x$ is defined as the impact parameter direction on the reaction plane. Sideward flow is generated mainly by the participant-spectator interaction. Nucleons in the projectile feel repulsive interaction from the target nucleus during the projectile-target contact time. This repulsion pushes projectile nucleons out in the positive sideward direction if the contact time is long enough. When the incident energy is very high, the contact time in collisions becomes shorter owing to the Lorentz contraction; therefore sideward flow decreases. At SPS energies mainly other types of collective flows, called directed $\left(v_{1}\right)$ and elliptic $\left(v_{2}\right)$ flows, are measured. These are defined as the $n$th Fourier coefficient,

$$
\frac{d^{3} N}{p_{T} d p_{T} d y d \phi}=\frac{d^{2} N}{2 \pi p_{T} d p_{T} d y}\left(1+\sum_{n} 2 v_{n}\left(p_{T}, y\right) \cos n \phi\right),
$$

where the azimuthal angle $\phi$ is measured from the reaction plane. The directed flow $v_{1}$ is the first Fourier coefficient of the azimuthal distribution

$$
v_{1}=\langle\cos \phi\rangle=\left\langle\frac{p_{x}}{p_{T}}\right\rangle,
$$

and the elliptic flow $v_{2}$ is the second Fourier coefficient of the azimuthal distribution

$$
v_{2}=\langle\cos 2 \phi\rangle=\left\langle\frac{p_{x}^{2}-p_{y}^{2}}{p_{T}^{2}}\right\rangle .
$$

These collective flows are reviewed in Ref. [57].

The effects of the MF in high-energy heavy-ion collisions are visible but not very large in single-particle spectra, such as the rapidity distribution $d N / d y$ or the transverse mass distribution $d^{2} N / m_{T} d m_{T} d y$. In this section we demonstrate that MF effects are essential for studying anisotropic collective flows in the hadron-string transport model JAM with MF potentials.

\section{A. Collective flows at AGS energies}

We show proton sideward flow $\left\langle p_{x}\right\rangle$ in mid-central $\mathrm{Au}+\mathrm{Au}$ collisions at AGS energies $\left[E_{\mathrm{inc}}=(2-11) A \mathrm{GeV}\right]$ together with AGS-E895 data [20] in Fig. 2 and in the left-hand panel of Fig. 3 . We choose the impact parameter range $4<b<8 \mathrm{fm}$ in the calculations, which roughly corresponds to mid-central collisions in experimental data.

It is seen that both cascade and momentum-independent soft (S) MF results are inconsistent with the data. The magnitude
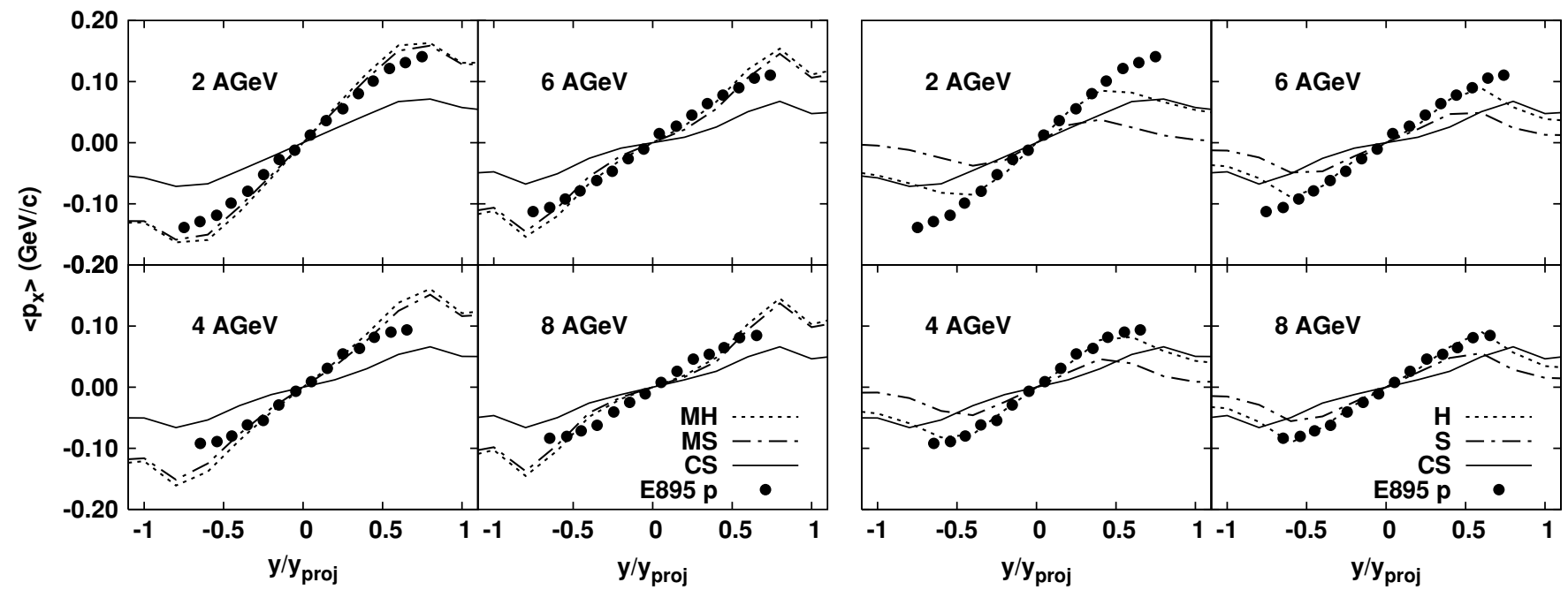

FIG. 2. Sideward flows $\left\langle p_{x}\right\rangle$ of protons in mid-central Au+Au collisions at (2-8)A GeV are compared with the AGS-E895 data [20]. Curves show the calculated results of cascade with momentum-dependent hard or soft mean field (MH or MS, left-hand panels), cascade with momentum-independent mean field (H or S, right-hand panels), and cascade without mean field (CS). The experimental data are shown in both the left and the right panels. 



FIG. 3. Comparison of calculated sideward flow $\left\langle p_{x}\right\rangle$ of protons (left) and pions (right) in $11 A \mathrm{GeV} \mathrm{Au}+\mathrm{Au}$ mid-central collisions to AGS-E877 data [18]. The meaning of the curves is the same as in Fig. 2.

of $\left\langle p_{x}\right\rangle$ in the forward rapidity region $\left(y / y_{\text {proj }} \simeq \pm 1\right)$ is small compared with the data, and the slope parameters at mid-rapidity are also smaller than that of the data with soft MF. The momentum-independent soft MF reduces $\left\langle p_{x}\right\rangle$ in the forward rapidity region and enhances the slope parameters at mid-rapidity. The former is an unfavorable effect in explaining the data, and the latter is not enough. With momentum-independent hard $(\mathrm{H}) \mathrm{MF}$, the slope parameter is well reproduced, but the $\left\langle p_{x}\right\rangle$ at forward rapidities are smaller than the data, especially at $E_{\text {inc }}=2 A$ and $11 \mathrm{~A} \mathrm{GeV}$.

Proton sideward flow data are qualitatively reproduced with the momentum-dependent MF. The momentum-dependent MF pushes up the flow almost linearly as a function of rapidity, and it becomes closer to the data, while the $\left\langle p_{x}\right\rangle$ values at forward rapidities may be a little too large compared with the data at $E_{\mathrm{inc}}=(4-8) A \mathrm{GeV}$. As the incident energy increases, MF effects on the slope parameter at mid-rapidity become small, but we can still see clear differences at forward rapidities between the results with and without momentum dependence.

Our results suggest the necessity of the momentum dependence in the MF to yield large-magnitude emission in the $x$ direction at forward rapidity. We note that our results with momentum-dependent MF are consistent with the previous calculations with MF on the collective flow data at AGS energies [25,28,33] as well as SIS energies [15,30].

The importance of momentum dependence in the MF is also seen in the transverse momentum dependence of the proton $v_{2}$ as shown in Fig. 4. Only if momentum dependence is included do we reproduce the strong squeezing at $E_{\mathrm{inc}}=$ $2 A \mathrm{GeV}$ of the $p_{T}$ dependence.

In the right-hand panel of Fig. 3, we plot the results of sideward flow $\left\langle p_{x}\right\rangle$ for pions in $\mathrm{Au}+\mathrm{Au}$ collisions at $E_{\mathrm{inc}}=$ $11 A \mathrm{GeV}$. The sideward flow $\left\langle p_{x}\right\rangle$ of pions are suppressed significantly by momentum-dependent MF. This may be because pions are trailed by nucleons, which is affected by MF, giving visible differences.

\section{B. Directed flow at SPS energies}

Directed flow $v_{1}$ has been measured at SPS energies [ $E_{\text {inc }}=$ $(40,158) A \mathrm{GeV}]$ instead of $\left\langle p_{x}\right\rangle$ as a function of rapidity. In Fig. 5, we compare the rapidity dependence of proton $v_{1}$ with the data in mid-central $\mathrm{Pb}+\mathrm{Pb}$ collisions at $E_{\text {inc }}=40 A$ and $158 \mathrm{~A} \mathrm{GeV}$ from the CERN-NA49 Collaboration [24], both of which are deduced by the reaction plane method (standard method). One can see that momentum-dependent MF generally improves the description of $v_{1}$.



FIG. 4. Transverse momentum dependence of the elliptic flow $v_{2}$ for protons in $\mathrm{Au}+\mathrm{Au}$ mid-central collisions at $(2,4,6) A \mathrm{GeV}$ are compared with AGS-E895 data [20]. The meaning of the curves is the same as in Fig. 2. 

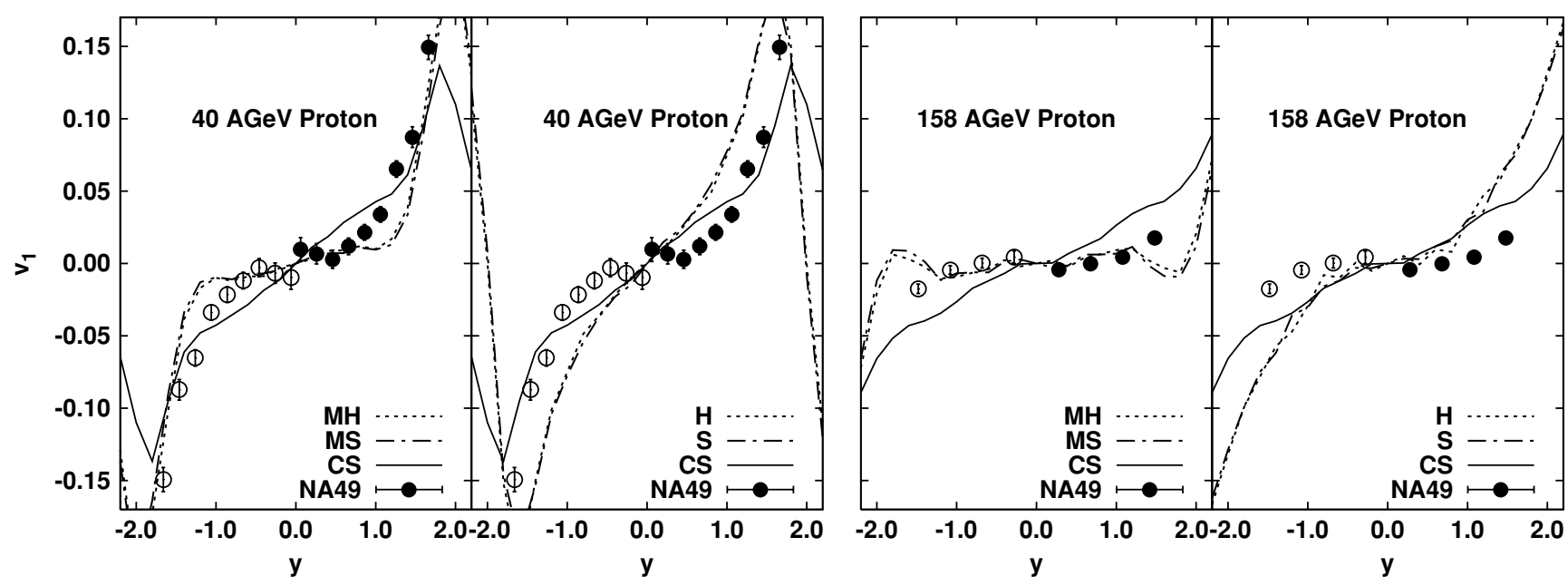

FIG. 5. Proton directed flows $v_{1}$ as a function of rapidity in mid-central $\mathrm{Pb}+\mathrm{Pb}$ collisions at $E_{\text {inc }}=40 A \mathrm{GeV}$ (left panel) and $158 A$ GeV (right panel) in comparison with SPS-NA49 data [24]. Curves show the calculated results of cascade with momentum-dependent hard or soft mean field (MH or MS), cascade with momentum-independent mean field (H or S), and cascade without mean field (CS).

It is interesting to note that the cascade model overestimates $v_{1}$ for protons, in contrast to the underestimate of $\left\langle p_{x}\right\rangle$ at AGS energies. We also see that $v_{1}$ is reduced at SPS energies with momentum-dependent MF, while $\left\langle p_{x}\right\rangle$ is enhanced at AGS energies. This behavior is the reverse of that at lower incident energies. Note also that the results with momentumindependent MF predict a larger $v_{1}$ than do the cascade results.

In Fig. 5 the results from the momentum-dependent MF show a flat behavior at mid-rapidity at $158 \mathrm{~A} \mathrm{GeV}$. The wiggle (a negative slope of the proton $v_{1}$ near mid-rapidity) [29] has been reported at peripheral collisions [24]. It would be interesting to study this in detail in the future.

In Fig. 6 we compare the transverse momentum dependence of $v_{1}$ for protons in $\mathrm{Pb}+\mathrm{Pb}$ collisions at $E_{\text {inc }}=40 A$ and $158 \mathrm{~A} \mathrm{GeV}$ with the data. We choose rapidity cut $|y|<1.8$ for $40 A \mathrm{GeV}$ and $|y|<2.1$ for $158 \mathrm{~A} \mathrm{GeV}$ according to the experimental cuts. The $p_{T}$ dependence at $158 \mathrm{~A} \mathrm{GeV}$ is very different from that at $40 \mathrm{~A} \mathrm{GeV}$. Dense baryonic matter is tentatively formed in the calculations up to around $40 \mathrm{~A} \mathrm{GeV}$, while many strings are formed and hadrons are formed later at $158 \mathrm{~A} \mathrm{GeV}$ at mid-rapidity. As a result, $v_{1}$ does not necessarily grow as a function of $p_{T}$ at $158 \mathrm{~A} \mathrm{GeV}$, because strings do not feel the MF in our model, and hadrons with large $p_{T}$ from string decay have a long formation time in the total CM system, and they would have smaller chances to interact with other hadrons before strings decay.

Let us now turn to the pion $v_{1}$. We show $v_{1}$ for pions as a function of rapidity in Fig. 7 and transverse momentum in Fig. 8 at $40 A$ and $158 A \mathrm{GeV}$. It is seen that MF effects for pion $v_{1}$ are very small, especially at mid-rapidities. MF effects are seen only in the forward rapidity region for momentum-dependent MF. At $40 \mathrm{~A} \mathrm{GeV}$, in the forward rapidity region, we find reduction (enhancement) of $v_{1}$ in the momentum-dependent (-independent) MF results compared with cascade ones. This comes from the counteraction from

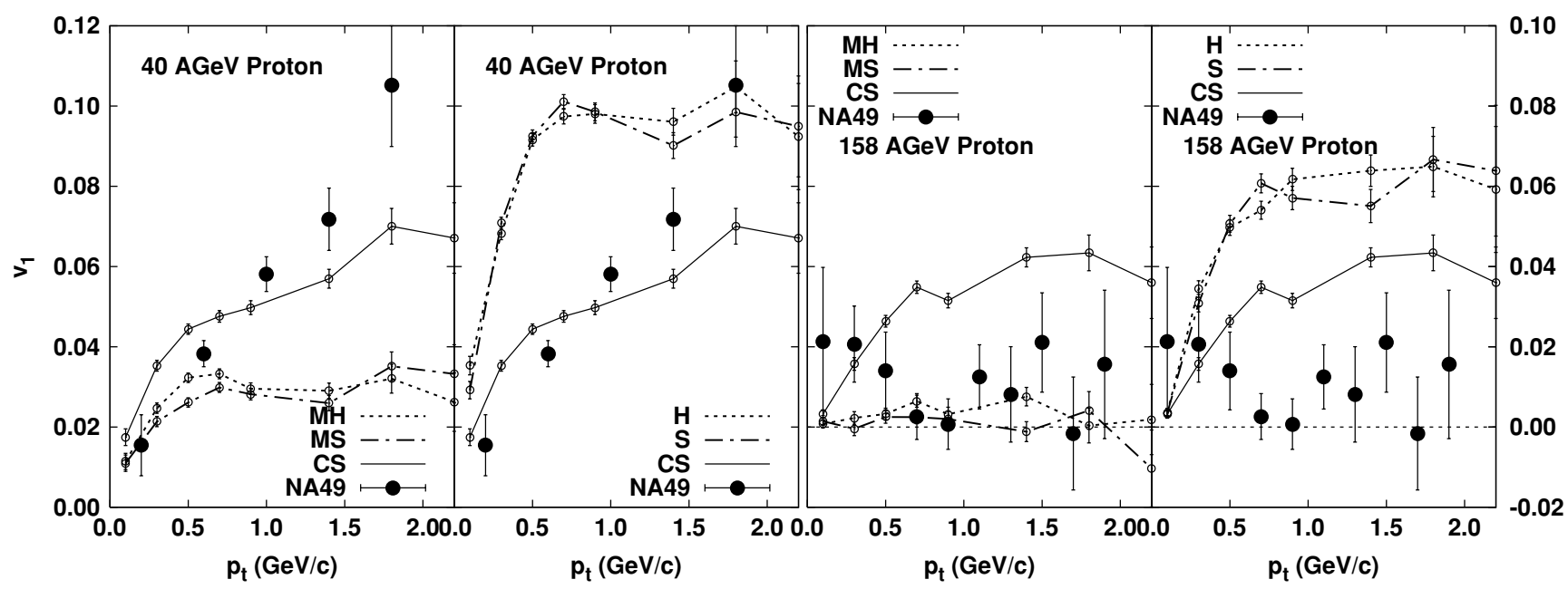

FIG. 6. Proton directed flows $v_{1}$ as a function of transverse momentum in mid-central $\mathrm{Pb}+\mathrm{Pb}$ collisions at $E_{\text {inc }}=40 A$ GeV (left panel) and $158 \mathrm{~A} \mathrm{GeV}$ (right panel) are compared with SPS-NA49 data [24]. The meaning of the curves is the same as Fig. 5. 




FIG. 7. Pion directed flows $v_{1}$ as a function of rapidity in mid-central $\mathrm{Pb}+\mathrm{Pb}$ collisions at $E_{\text {inc }}=40 \mathrm{~A} \mathrm{GeV}$ (left panel) and $158 \mathrm{~A}$ GeV (right panel) are compared with SPS-NA49 data [24]. The meaning of the curves is the same as Fig. 5.

protons; momentum-dependent (-independent) $\mathrm{MF}$ reduces (enhances) proton $v_{1}$ in the mid-rapidity region, and pion $v_{1}$ anticorrelates with proton $v_{1}$. Probably we need to include pion MF for a better understanding of the collective flows at SPS energies.

\section{Elliptic flow at SPS energies}

Since the $v_{1}$ signal becomes small owing to the short participant-spectator interaction time at high energies, the next Fourier coefficient, called the elliptic flow $v_{2}$, has been discussed more extensively at SPS and RHIC. At these energies, the participants form an almondlike shape in the transverse plane after the spectators go through, and these almond-shaped participants start to expand more strongly in the $x$ (shorter axis of the almond) direction because of the higher-pressure gradient if the participants are well thermalized. This expansion is known to lead to the enhancement of in-plane particle emission, i.e., positive elliptic flow $v_{2}$.

In Figs. 9 and 10 we plot the results of the rapidity and transverse momentum dependence of $v_{2}$ for protons at SPS energies ( $40 A$ and $158 A \mathrm{GeV}$ ) together with the SPS-NA49 data [24]. At SPS energies, the cascade model generally explains the proton $v_{2}$ data qualitatively, including the flat behavior of $v_{2}(y)$ at mid-rapidities at $158 A \mathrm{GeV}$ and the approximate linear $p_{T}$ dependence of $v_{2}\left(p_{T}\right)$. One exception is the missing collapse of $v_{2}(y)$ at mid-rapidity at $40 A \mathrm{GeV}$. This collapse seen in the NA49 data may be an indication of a first-order phase transition at high baryon densities achieved in the $\mathrm{Pb}+\mathrm{Pb}$ collisions at $40 A \mathrm{GeV}$ [34].

Effects of MF are small for proton $v_{2}$ at SPS energies. Elliptic flow is most easily generated in the early stages of the collisions, since spatial anisotropy is the largest. However, at

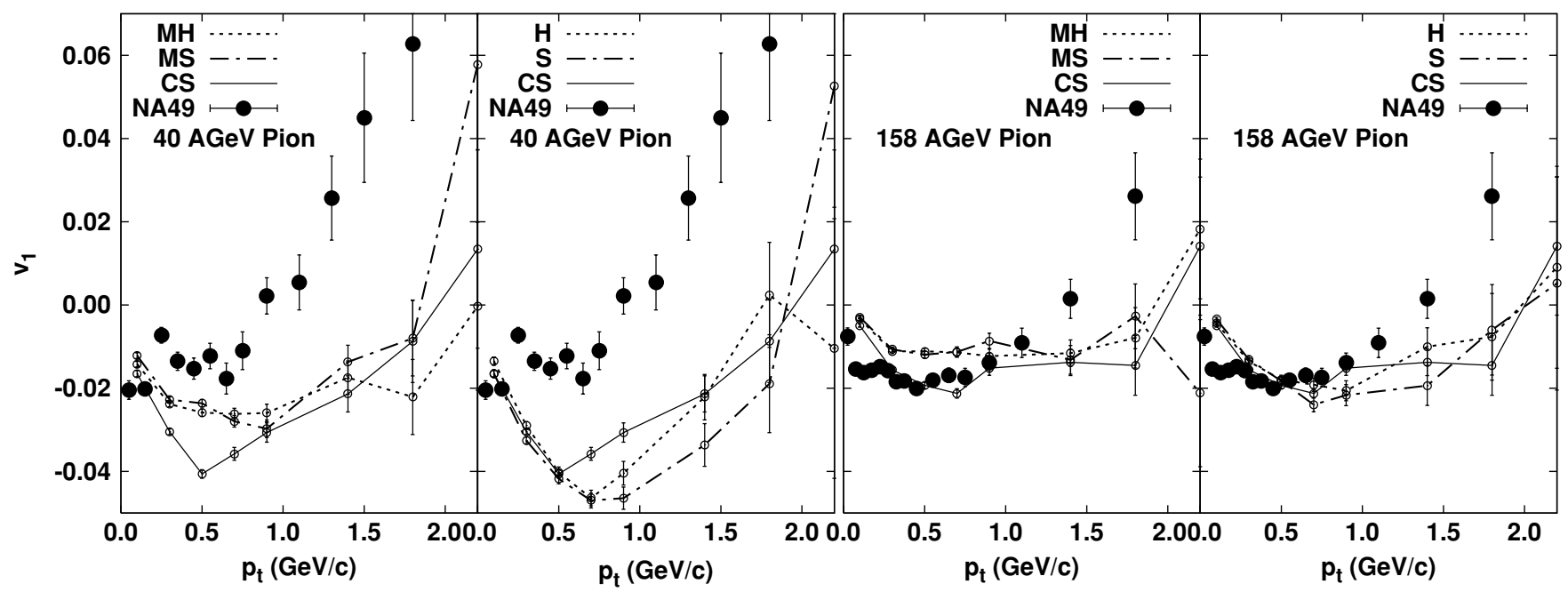

FIG. 8. Pion directed flows $v_{1}$ as a function of transverse momentum for $0<y<1.5$ in $\mathrm{Pb}+\mathrm{Pb}$ collisions at $E_{\text {inc }}=40 A$ GeV (left panel) and $158 \mathrm{~A} \mathrm{GeV}$ (right panel) are compared with SPS-NA49 data [24]. The meaning of the curves is the same as Fig. 5. 




FIG. 9. Proton elliptic flows $v_{2}$ as a function of rapidity in mid-central $\mathrm{Pb}+\mathrm{Pb}$ collisions at $40 \mathrm{~A} \mathrm{GeV}$ (left) and $158 \mathrm{~A} \mathrm{GeV}$ (right). Lines show the calculated results of cascade with momentum dependent hard or soft mean field (MH or MS), cascade with momentum-independent mean field (H or S), and cascade without mean field (CS). Experimental data are taken from SPS-NA49 [24].

SPS energies, string excitations dominate particle production at early times in the model, and those strings are not affected by the nuclear mean field. That is the reason that MF effects are small at SPS energies in our results.

The rapidity and the transverse momentum dependences of the pion $v_{2}$ are shown in Figs. 11 and 12, respectively. The rapidity dependence at $158 \mathrm{~A} \mathrm{GeV}$ and the transverse momentum dependence at low $p_{T}\left(p_{T}<1 \mathrm{GeV} / c\right)$ at $40 A$ and $158 \mathrm{~A} \mathrm{GeV}$ are well explained by the cascade model as well as by the momentum-dependent or -independent MF models, and we do not find any significant MF effects for these observables. By contrast, we do not see the collapse of $v_{2}(y)$ at mid-rapidities seen in the $40 \mathrm{~A} \mathrm{GeV} \mathrm{NA49} \mathrm{data,} \mathrm{and}$ we underestimate $v_{2}$ at high $p_{T}$. The former corresponds to the collapse of proton $v_{2}(y)$ mentioned before. Momentumindependent MF enhances pion $v_{2}(y)$ slightly, but this is in the reverse direction needed to explain the data at $40 \mathrm{~A} \mathrm{GeV}$. The strong increase of $v_{2}\left(p_{T}\right)$ up to around $p_{T} \sim 2 \mathrm{GeV} / c$ is also seen at RHIC energies, and this behavior is discussed as an indication of hydrodynamic evolution [50].

We now turn to the discussion of the difference between $v_{1}$ and $v_{2}$. We have shown that MF effects on $v_{1}$ are rather strong, but $v_{2}$ is relatively insensitive to MF at SPS energies. This may come from the difference of developing time between $v_{1}$ and $v_{2}$. The directed flow $v_{1}$ at mid-rapidities is generated mainly by the interaction between participants and spectators in the early stage of the collision, where baryon density is the highest. On the other hand, $v_{2}$ in our model is generated in the late stage until the time reaches the order of the nuclear radius, where densities are not very high, but it is not formed in the early stage in our model. This is because our current hadronic transport approach does not have large participant

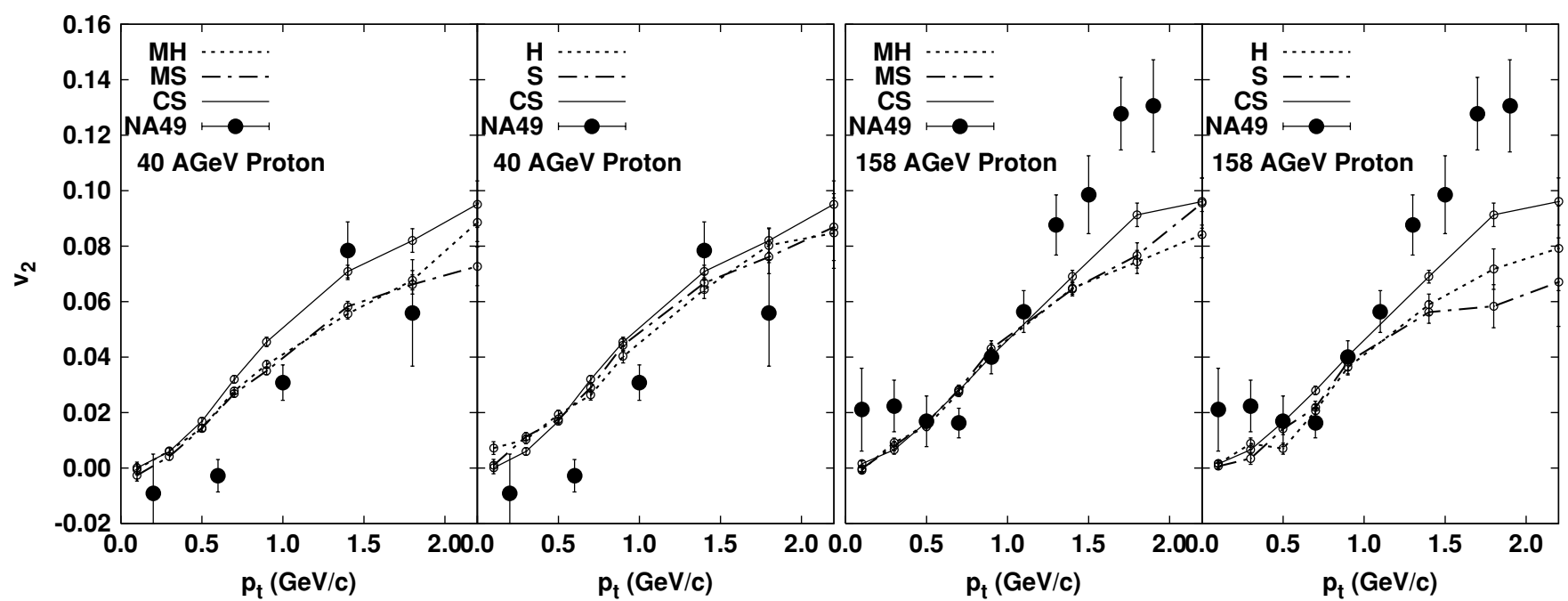

FIG. 10. Proton elliptic flows $v_{2}$ as a function of transverse momentum. The meaning of the curves is the same as Fig. 9. 


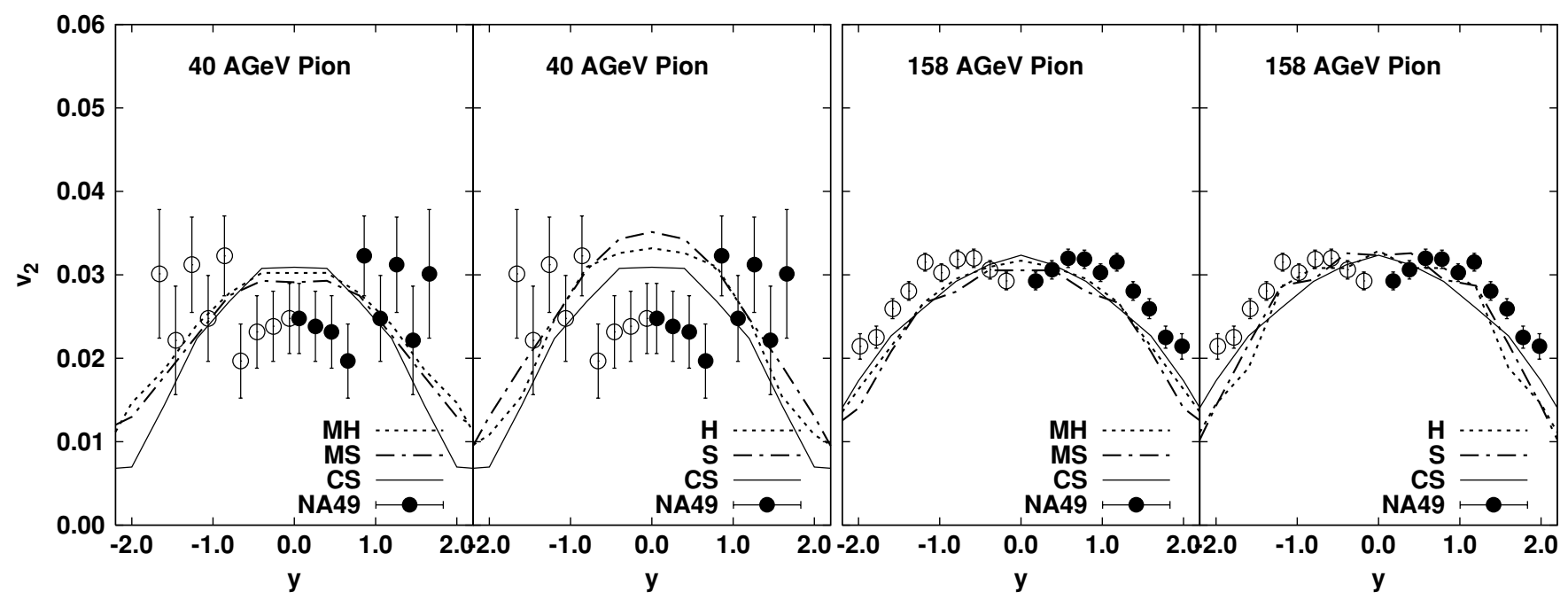

FIG. 11. Pion elliptic flows $v_{2}$ as a function of rapidity. The meaning of the curves is the same as Fig. 9.

pressure in the early stages of the collisions, as we do not explicitly include MF for strings and partonic interactions. In a hydrodynamic picture, $v_{2}$ develops from very early times owing to thermal pressure. This is a striking difference between our approach and hydrodynamics, as was previously studied in Ref. [41].

\section{Elliptic flow excitation functions from AGS to SPS energies}

When the incident energy is not high enough, spectators squeeze participants out of the reaction plane owing to the repulsive nuclear interactions at $0.2 A \lesssim E_{\text {inc }} \lesssim 4 A \mathrm{GeV}$. This squeezing leads to a negative value of the elliptic flow of nucleons $\left(v_{2}<0\right)$. The elliptic flow, therefore, shows the strength of the repulsive interaction at lower energies. On the other hand, elliptic flow becomes positive at higher energies, because there is no such squeezing effect that is due to the Lorentz contraction. Elliptic flow gives a information how much pressure is generated at higher energies.
In Fig. 13 we show the incident energy dependence of proton $v_{2}$ in mid-central collisions with measured data $[-0.1<y<0.1$ for AGS, $0<y<2.1(0<y<1.8)$ for SPS $158 A(40 A) \mathrm{GeV}][19,24]$. Rapidity cut $|y|<0.2 y_{\text {proj }}$ has been used in the calculations. Experimental data clearly show the evolution from squeezing to almond-shaped participant dynamics. With both cascade and momentum-independent soft MF (S), we cannot explain strong squeezing effects at lower energies. The calculated $v_{2}$ values for momentum-independent $\operatorname{MF}(\mathrm{H}, \mathrm{S})$ and cascade are generally larger than data at AGS energies. Momentum-dependent MF (MH, MS), which is repulsive in the incident energy range under consideration, pushes down the elliptic flow significantly. We qualitatively reproduce the incident energy dependence from AGS [19] to SPS [24] energies.

Calculated results with both $\mathrm{MH}$ and MS are smooth as a function of beam energy, while the data at $E_{\text {inc }}=40 A \mathrm{GeV}$ has a dip [24]. Confirmation of data is necessary to examine the incident energy dependence of $v_{2}$, whether it is a monotonic

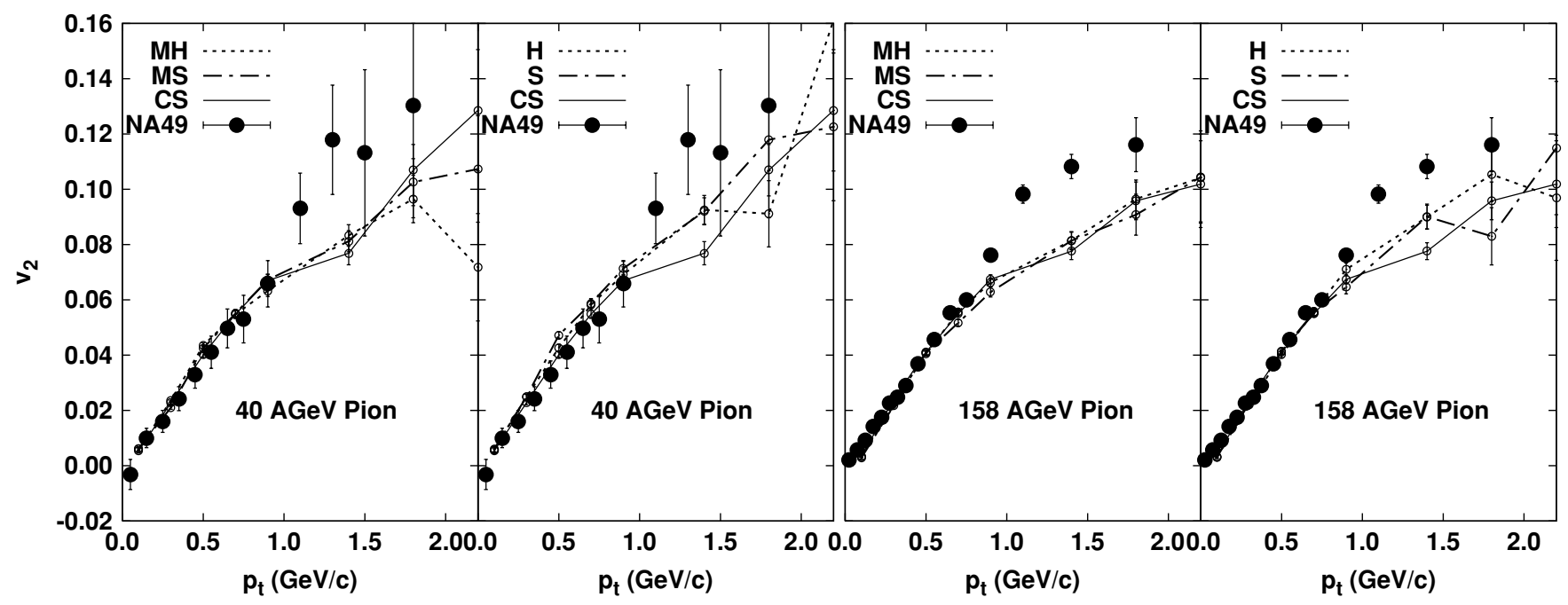

FIG. 12. Pion elliptic flow $v_{2}$ as a function of transverse momentum. The meaning of the curves is the same as Fig. 9. 


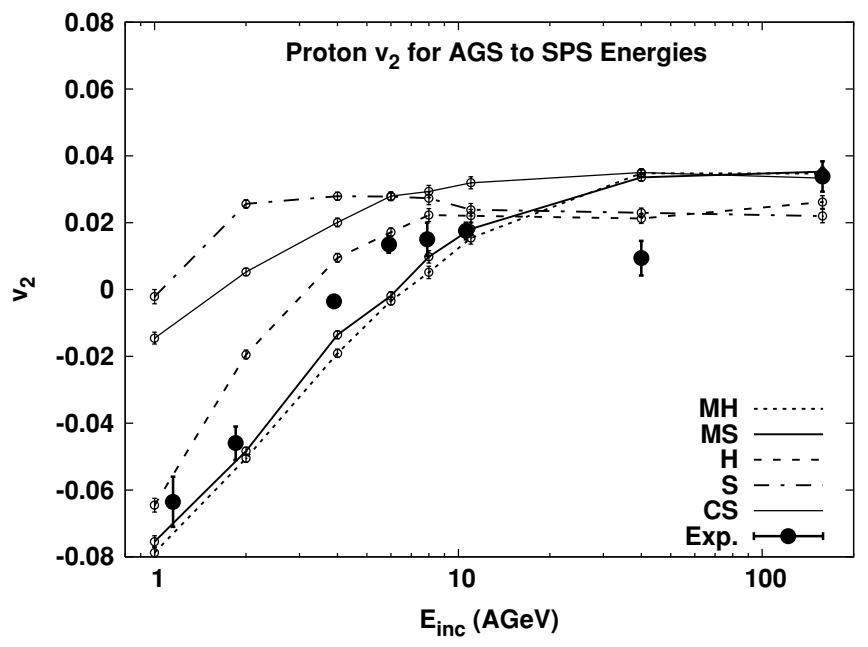

FIG. 13. Incident energy dependence of proton elliptic flow at mid-rapidities in mid-central heavy-ion collisions from $1 A \mathrm{GeV}$ to $158 A \mathrm{GeV}$. Dotted, bold-solid, dashed, and dotted-dashed and thinsolid lines show the results of cascade with MH, MS, H, S mean-field, and cascade without mean-field (CS), respectively. The experimental data are taken from LBL-EOS, AGS-E895, E877 from Ref. [19], and SPS-NA49 from Ref. [24].

function or has a dip at around $E_{\text {inc }} \sim 40 A \mathrm{GeV}$, by looking at the missing data points.

In our results with momentum-dependent potentials, the stiffness dependence of $v_{2}$ is smaller than that in the Boltzmann equation model (BEM) [19,27,28]. In the RQMD/S framework with the relativistic distance $\tilde{\boldsymbol{r}}_{i j}^{2}$, the interaction between the projectile and target nucleons are suppressed at high energies by the factors $m_{i} / p_{i}^{0}$ and $m_{j} / p_{j}^{0}$ in the potential derivatives in Eqs. (A25) and (A26). For momentum-dependent potentials, we have the relative momentum vector $\boldsymbol{p}_{i j}$ in Eq. (A29), which can compensate for the suppression factor in Eq. (A26). For momentum-independent potentials, on the other hand, the pair velocity $\boldsymbol{\beta}_{i j}$ in Eq. (A20) is very small for nucleon pairs between the projectile and target, and there is no enhancement factor to compensate for the above suppression in the derivatives of the relativistic distance $\tilde{\boldsymbol{r}}_{i j}^{2}$ in Eqs. (A27) and (A28). This suppression does not happen in BEM, and they find significant stiffness dependence in Refs. [19,27,28], while we do not see strong stiffness dependence. In the case of momentum-independent potentials, our results are closer to the cascade results compared with those in Refs. [48,58]. This difference also comes from the above suppression between the projectile and target nucleons. The essential reason for these differences is that in $\mathrm{RQMD}$ or $\mathrm{RQMD} / \mathrm{s}$ potentials are regarded as Lorentz scalar. Possible other model dependences will be discussed in the next section.

\section{MODEL UNCERTAINTIES}

In the previous section it has been shown that the momentum-dependent hard or soft MF improved the description of the collective flow data from AGS to SPS energies. However, there are some uncertainties in our calculations for the study of collective flows.

First, let us consider the effects of the MF for nonnucleonic baryons. Strange baryons, resonance hadrons, or antibaryons are expected to feel MF, which may be different that affecting nucleons. In the previous section we assumed that all the baryons feel the same MF, and this treatment would give a rough estimate of a maximum $\mathrm{MF}$ effect, since, for example, the MF for $\Delta$ 's or $\Lambda$ 's is generally expected to be smaller than that for nucleons. On the other hand, if we include MF only for nucleons, we may get a rough estimate of a minium baryonic MF effects.

In Figs. 14 and 15, we compare the results with and without MF for nonnucleonic baryons. We can read in the left-hand panel of Fig. 14 that ignoring MF for nonnucleonic baryons (specified as $\mathrm{N}$ in the figure) at $2 A \mathrm{GeV}$ reduces both the $\left\langle p_{x}\right\rangle$ slope and the strength at the forward rapidities by about $20 \%$ compared with the case of $\mathrm{B}$, in which all baryons feel MF. But the slope remains the same at $11 A \mathrm{GeV}$ for $\mathrm{N}$ and $\mathrm{B}$. On the other hand, it is seen in the right-hand panel of Fig. 14
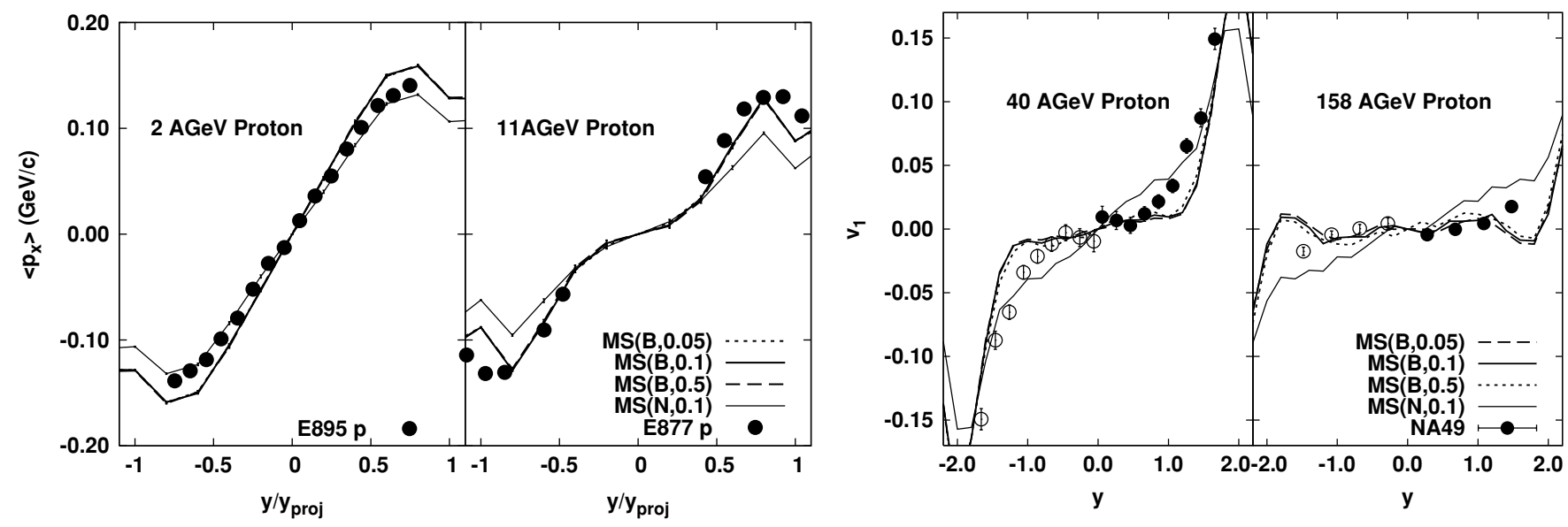

FIG. 14. Sideward (directed) flows of AGS (left) and SPS (right) energies are compared with different assumptions for the mean fields. The momentum-dependent soft (MS) mean-field has been used in these calculations. The first term in the parenthesis means that all baryons (B) or only nucleons $(\mathrm{N})$ are included for the MF. The second term $(0.05 / 0.1 / 0.5)$ denotes simulation time step size $d t(\mathrm{fm} / c)$. 


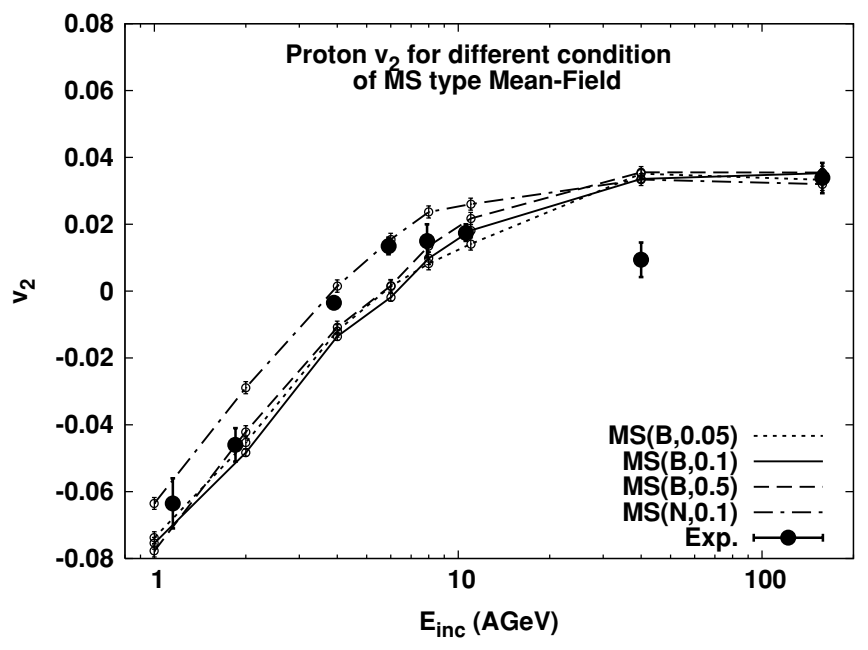

FIG. 15. Elliptic flows are compared with different assumptions for the mean fields. The meaning of the curves is the same as Fig. 14.

that MF only for nucleons is not enough to suppress $v_{1}$ at SPS energies. One can also see some differences of $v_{2}$ in B and $\mathrm{N}$ in Fig. 15 up to AGS energies. The experimental data lie between $\mathrm{B}$ and $\mathrm{N}$ except for $40 \mathrm{~A} \mathrm{GeV}$, suggesting that the MF for excited baryons is smaller than that for nucleons.

Next, we have checked the time step size $d t$ dependence. Since the update of MF after each collision requires a huge calculation time, we evaluate MF only at each time slice. When a baryon collides in one time step, that baryon is propagated with MF until the collision time, and we ignore the MF after the collision before it is formed. In the time step of baryon formation, displacements by the MF for $\boldsymbol{p}_{i}$ and $\boldsymbol{r}_{i}$ are evaluated by using the MF at the next time slice. This treatment is valid up to the first order in $d t$ when one baryon collides or is formed once in one time step. In the later stages this prescription is expected to work well because of the low collision frequency. In the early stages many collisions make strings and resonances, which do not feel MF, so our prescription may not be too bad. In the middle stages, however, it may be possible that elastic scatterings are frequent enough and baryons continue to feel MF after collisions in each time step. Thus we need to analyze the collision frequency effects on constructing flows by reducing $d t$. In Figs. 14 and 15 we plot the results with different time step sizes $d t=0.05,0.1$, $0.5 \mathrm{fm} / c$. For $\left\langle p_{x}\right\rangle$ at AGS energies (left-hand panel of Fig. 14), all the results with different time step size agree well with each other, and we cannot distinguish these lines. The time step size dependence of $v_{2}$ as shown in Fig. 15 still gives us confirmation of the convergence of the numerical results. For $v_{1}$ at SPS energies (right-hand panel of Fig. 14), only very small differences can be seen between the results with $d t=0.5 \mathrm{fm} / c$ and $d t \leqslant 0.1 \mathrm{fm} / c$. We conclude that $d t=$ $0.1 \mathrm{fm} / c$, which has been used as a default throughout this work, is small enough to perform a reliable calculations.

Finally, we would like to address the problem of the uncertainties of the transport model itself. In addition to the ambiguities in introducing collision terms, the equations of motion depend on the model treatment. It is not trivial at all to construct equations of motion of relativistic particles during heavy-ion collisions based on the potential or the MF, giving an appropriate EOS. At relativistic energies there are proposed several ways to introduce the potential effects.

(i) Relativistic mean field (RMF), having Lorentz scalar $U_{s}$ and vector $U_{v}^{\mu}$ terms (RBUU [25,32]). The scalar and the vector time component are evaluated in the local rest frame, and by the Lorentz transformation we can get $U_{v}^{\mu}$ in the calculation frame. Thus, neglecting a nonlocality in time, this evaluation of the potential is practically covariant. In this approach, however, we need to introduce strong cutoff for the coupling of vector meson and baryons [25], since the vector potential effects linearly increase as a function of incident energy.

(ii) Lorentz scalar reinterpretation of nonrelativistic potentials (BUU [31], BEM [27]). In the BUU model [31] the Lorentz scalar MF $U_{s}$ is obtained from the nonrelativistic MF $U$ in the local rest frame through the relation

$$
\begin{aligned}
\varepsilon(\boldsymbol{p}, \rho) & =\sqrt{\left[m+U_{s}(\boldsymbol{p}, \rho)\right]^{2}+\boldsymbol{p}^{2}} \\
& =\sqrt{m^{2}+\boldsymbol{p}^{2}}+U(\boldsymbol{p}, \rho),
\end{aligned}
$$

where $\rho$ is the baryon density [31]. For the momentumindependent MF in BEM in Refs. $[27,28]$, the scalar potential is directly given so as to fit the EOS, and the scalar density is used for $\rho$. They do not have any vector terms increasing at high energies, and the potential effects become mild compared with the RMF treatment. For example, the derivative of the above single-particle energy gives rise to the factor $\left(m+U_{s}\right) / \varepsilon$ in front of the $U_{s}$ derivative and suppresses the potential effects.

(iii) Combination of the Lorentz scalar and nonrelativistictype density-dependent potentials (BEM [26-28]). This approach is adopted in Ref. [26] and in the momentumdependent MF in Refs. [27,28]. The single-particle energy is given as

$$
\begin{aligned}
\varepsilon(p, \rho) & =m+\int_{0}^{p} d p^{\prime} v^{*}\left(p^{\prime}, \rho\right)+\widetilde{U}(\rho), \\
v^{*}(p, \rho) & =\frac{p}{\sqrt{p^{2}+\left[m^{*}(p, \rho)\right]^{2}}} .
\end{aligned}
$$

The derivative of $\widetilde{U}(\rho)$ does not come with a suppression factor such as $m / \varepsilon$, and it generates strong effects at high energies, where both the density and the density derivative become large.

(iv) Constraint Hamiltonian dynamics (RQMD [39], RQMD/s [43]). In RQMD and RQMD/s, particle velocity and force are not given by the derivatives of the single-particle energy but by the derivatives of the total Hamiltonian, as shown in the Appendix in the case of RQMD/s. Thus the relation to other MF models described above is not straightforward. However, the potential $V_{i}$ in the on-massshell constraint (A8) is introduced as Lorentz scalar, and we have suppression factor $m / p^{0}$ in the equations of motion, (A14) and (A15). These observations suggest that the RQMD and RQMD/S would give results similar to those in Lorentz scalar MF models, such as BUU [31]. Another difference from other MF models exists in the 
nuclear density profile. One nucleon is represented by one Gaussian packet rather than many test particles, then the nuclear diffuseness becomes generally larger in QMDtype models. This may generate artificial surface effects at large impact parameters or in light-ion collisions. However, central and mid-central collisions of heavynuclei are expected to be well described, as in the case of various cascade models, in which one particle is used for one hadron.

We are not very sure which is the best way to include the potential effects in high-energy heavy-ion collisions. Further formal developments on transport models in relation to the nuclear EOS would be necessary, and at the same time, phenomenological studies of heavy-ion collisions are required in order to verify the validity of models and to elucidate the EOS. From the latter point of view, systematic study in wide range of incident energy is needed, since the above uncertainties are closely related to the Lorentz transformation properties, whose effects would vary drastically as the incident energy varies. The incident energy range from AGS to SPS energies studied in this work may provide a good benchmark test for transport models and the EOS.

\section{SUMMARY}

We have investigated collective flows in heavy-ion collisions from AGS $[(2-11) A \mathrm{GeV}]$ to $\operatorname{SPS}[(40,158) A \mathrm{GeV}]$ energies by using a combined framework of hadron-string cascade (JAM) [49] and covariant constraint Hamiltonian dynamics (RQMD/S) [43]. In JAM, various particle production mechanisms are taken into account - production and decay of resonances and strings, jet production, and its fragmentation. Momentum dependence of the MF is fitted [55] to the real part of the Schrödinger equivalent global optical potential of Hama et al. [38] in a Lorentzian form, Eq. (1). Saturation properties are fitted by introducing the density-dependent potential of the Skyrme type in the power series of $\rho$. Calculated results of cascade, cascade with momentum-dependent MF, and cascade with momentum-independent MF are compared with the data of sideward $\left\langle p_{x}\right\rangle$, directed $v_{1}$, and elliptic $v_{2}$ flows as a function of rapidity, transverse momentum, and beam energy from AGS to SPS. Generally, results with momentum-dependent MF explain the trend of the data for proton flows reasonably well. We note that this is the first that the anisotropic proton collective flow data of heavy-ion collisions from AGS to SPS has been explained in one framework consistently. Without momentum dependence in $\mathrm{MF}$, we cannot reproduce the strong enhancement of the sideward flow at $E_{\text {inc }}=(2-11) A \mathrm{GeV}$, strong squeezing seen in $v_{2}$ for $E_{\text {inc }} \lesssim 4 A \mathrm{GeV}$, and the suppression of proton $v_{1}$ at $E_{\text {inc }}=40 A$ and $158 A \mathrm{GeV}$.

Our new model - a hadron-string cascade with momentumdependent $\mathrm{MF}$ - provides an improved description for collective flows in mid-central collisions from AGS to SPS energies. The present analysis implies that the effects of the momentum-dependent potential is large up to the SPS energies.

There are still many problems in the attempt to pin down the equation of state of dense nuclear matter from heavy-ion data. First, we have made an assumption that the MF is taken into account only for baryons and that all the baryons feel the same MF. It would be interesting to extend the present work to discuss the MF effects for mesons and different MFs for hyperons and resonance hadrons and to look at the $\Lambda$ or kaon flow data. Second, we cannot make the soft EOS ( $K \sim$ $200 \mathrm{MeV}$ ) with the present form of the MF be consistent with the optical potential. When the momentum dependence is fitted to the optical potential by Hama et al. [38] in the Lorentzian form, the EOS necessarily becomes relatively stiff in combination with the Skyrme-type density-dependent form, as shown in Table I. The small sensitivity of the EOS to momentum in this work may suggest that the probed EOS range is not wide enough. Finally, the model dependence of the MF treatment has to be removed in order to obtain model-independent EOS information. For this purpose it is necessary to test various MF treatments in one framework. In the present model, we need to modify the on-mass-shell constraint to include Lorentz vector potentials or potentials of other types. It can be a breakthrough for a transport model-independent discussion of the EOS.

\section{ACKNOWLEDGMENTS}

We are grateful to Professor Tomoyuki Maruyama and Professor Pawel Danielewicz for useful discussions and comments. This work is supported in part by the Ministry of Education, Science, Sports and Culture, Grand-in-Aid for Scientific Research (C)(2), no. 15540243, 2003.

\section{APPENDIX: RQMD/S FORMALISM}

Here we briefly summarize the RQMD/S formalism developed by Maruyama et al. in Ref. [43] for completeness. The original RQMD formalism is initiated by Sorge et al. in Ref. [39]. RQMD(/S) is based on the constrained Hamiltonian dynamics [59], which is formulated in a manifestly covariant way. We use four-vectors $q_{i}^{\mu}$ and $p_{i}^{\mu}$ for the description of the $N$ particle system. Therefore we need to have $2 N$ constrains $\phi_{i}(i=1, \ldots, 2 N)$, as physical phase space is $6 N$ dimensional. Now our Hamiltonian may be constructed from the constraints $\phi_{i}$, and the Lagrange multiplier $u_{i}$ from the Dirac's constraint Hamiltonian formalism:

$$
H=\sum_{i=1}^{2 N-1} u_{i} \phi_{i}
$$

The equations of motion are then

$$
\begin{aligned}
& \frac{d q_{i}}{d \tau}=\left\{H, q_{i}\right\} \approx \sum_{j=1}^{2 N-1} u_{j} \frac{\partial \phi_{j}}{\partial p_{i}}, \\
& \frac{d p_{i}}{d \tau}=\left\{H, p_{i}\right\} \approx-\sum_{j=1}^{2 N-1} u_{j} \frac{\partial \phi_{j}}{\partial q_{i}},
\end{aligned}
$$

where the Poisson brackets are defined as

$$
\{A, B\} \equiv \sum_{k, \mu}\left(\frac{\partial A}{\partial q_{k}^{\mu}} \frac{\partial B}{\partial p_{k \mu}}-\frac{\partial A}{\partial p_{k \mu}} \frac{\partial B}{\partial q_{k}^{\mu}}\right),
$$




$$
\begin{aligned}
\left\{q_{i}^{\mu}, p_{j \nu}\right\} & =\delta_{\nu}^{\mu} \delta_{i j}, \quad\left\{q_{i}^{\mu}, q_{j}^{\nu}\right\}=0, \quad\left\{p_{i \mu}, p_{j \nu}\right\}=0, \\
i, j, k & =1, \ldots, N, \quad \mu, \nu=0,1,2,3,
\end{aligned}
$$

and the sign $\approx$ means the weak equality initiated by Dirac [60]. When we require that constraints $\phi_{i}$ should be conserved in time, then they fulfill

$$
\frac{d \phi_{i}}{d \tau}=\frac{\partial \phi_{i}}{\partial \tau}+\sum_{j=1}^{2 N-1} u_{j}\left\{\phi_{i}, \phi_{j}\right\} \approx 0 .
$$

Particle trajectories in $6 N$ phase space is uniquely determined by the equations of motion Eqs. (A2) and (A3) together with Eqs. (A10) and (A11) when $2 N$ constraints are given.

We use the following $2 N$ constraints in RQMD/s

$$
\phi_{i} \equiv \begin{cases}H_{i} & i=1, \ldots, N \\ \chi_{i-N} & i=N+1, \ldots, 2 N .\end{cases}
$$

First $N$ constraints are the on-mass-shell constraints

$$
H_{i} \equiv p_{i}^{2}-m_{i}^{2}-2 m_{i} V_{i} \approx 0, \quad i=1, \ldots, N .
$$

Remaining $N$ conditions constrain the time fixation of the particles. In the original RQMD time fixation [39,40,42], the $N \times N$ matrix has to be solved numerically at each time step to deduce inverse matrices. Moreover, if particle production or annihilation occurs, the time fixation is violated, and a initial $q_{i}$ of produced particles satisfying the constraints and energy conservation has to be imposed.

Maruyama et al. introduced a simplified time fixation in $\mathrm{RQMD} / \mathrm{S}$ with the global time parameter $\tau$ in Ref. [43], as

$$
\begin{aligned}
& \chi_{i} \equiv \hat{a} \cdot\left(q_{i}-q_{N}\right) \approx 0, \quad i=1, \ldots, N-1, \\
& \chi_{N} \equiv \hat{a} \cdot q_{N}-\tau \approx 0,
\end{aligned}
$$

where $\hat{a}$ is a four-component vector corresponding to $(1, \mathbf{0})$ at the rest frame of the particle and $q_{i}$ is space-time coordinate of the $i$ th particle. The constraints equation (A9) can be satisfied in the case of particle production.

Since the constraint $\phi_{i}(i=1, \ldots, 2 N-1)$ does not depend explicitly on $\tau$, the Lagrange multiplier $u_{i}(\tau)$ can be solved as

$$
u_{i} \approx-\frac{\partial \phi_{2 N}}{\partial \tau} C_{2 N, i}, \quad i=1, \ldots, 2 N-1,
$$

where

$$
C_{i j}^{-1} \equiv\left\{\phi_{i}, \phi_{j}\right\}, \quad i, j=1, \ldots, 2 N .
$$

The matrix $C$ (inverse of matrix $C^{-1}$ ) must exist, because we only allow for the $\tau$-dependent $2 N$ th constraint functions, which are combined with the $2 N-1$ constraints. Furthermore, $C$ can be obtained analytically, if we replace $p_{i}^{0}$ from the potential $V_{i}$ with the kinetic energy $\sqrt{\boldsymbol{p}_{i}^{2}+m_{i}^{2}}$. This is a great advantage from the point of view of CPU time. One obtains the RQMD/s Hamiltonian

$$
H \approx \sum_{i=1}^{N} u_{i}\left(p_{i}^{2}-m_{i}^{2}-2 m_{i} V_{i}\right),
$$

where

$$
u_{i}=\frac{1}{2 p_{i}^{0}}, \quad p_{i}^{0}=\sqrt{\boldsymbol{p}_{i}^{2}+m_{i}^{2}+2 m_{i} V_{i}} .
$$

The equations of motion are then

$$
\begin{aligned}
& \frac{d \boldsymbol{r}_{i}}{d \tau} \approx-\frac{\partial H}{\partial \boldsymbol{p}_{i}}=\frac{\boldsymbol{p}_{i}}{p_{i}^{0}}+\sum_{j=1}^{N} \frac{m_{j}}{p_{j}^{0}} \frac{\partial V_{j}}{\partial \boldsymbol{p}_{i}}, \\
& \frac{d \boldsymbol{p}_{i}}{d \tau} \approx \frac{\partial H}{\partial \boldsymbol{r}_{i}}=-\sum_{j=1}^{N} \frac{m_{j}}{p_{j}^{0}} \frac{\partial V_{j}}{\partial \boldsymbol{r}_{i}} .
\end{aligned}
$$

In actual calculations, we have replaced $p_{i}^{0}$ with the kinetic energy $\sqrt{\boldsymbol{p}_{i}^{2}+m_{i}^{2}}$ in the denominators of Eqs. (A14) and (A15) after evaluating all the derivative terms for simplicity. This approximation would be valid in the relativistic energy region, where the kinetic energy is much larger than the potential $V_{i}$.

Relative distance $\boldsymbol{r}_{i j}=\boldsymbol{r}_{i}-\boldsymbol{r}_{j}$ and $\boldsymbol{p}_{i j}=\boldsymbol{p}_{i}-\boldsymbol{p}_{j}$ in the potentials should be replaced by the squared four-vector distance with a Lorentz scalar, as

$$
\begin{aligned}
& -q_{T i j}{ }^{2}=-q_{i j}{ }^{2}+\frac{\left(q_{i j} \cdot P_{i j}\right)^{2}}{P_{i j}{ }^{2}}, \\
& -p_{T i j}{ }^{2}=-p_{i j}{ }^{2}+\frac{\left(p_{i j} \cdot P_{i j}\right)^{2}}{P_{i j}{ }^{2}},
\end{aligned}
$$

where $p_{i j}=p_{i}-p_{j}, q_{i j}=q_{i}-q_{j}, \quad P_{i j}=p_{i}+p_{j} . \quad$ We note that in the nonrelativistic limit $-q_{T i j}{ }^{2} c \rightarrow \infty r_{i j}^{2}$. This assumption takes into account the contraction of longitudinal direction, and we can avoid unphysical compression. In the actual simulations, we use the following expression:

$$
\begin{aligned}
& -q_{T i j}^{2} \equiv \tilde{\boldsymbol{r}}_{i j}^{2}=\boldsymbol{r}_{i j}^{2}+\gamma_{i j}^{2}\left(\boldsymbol{r}_{i j} \cdot \boldsymbol{\beta}_{i j}\right)^{2}, \\
& -p_{T i j}^{2} \equiv \tilde{\boldsymbol{p}}_{i j}^{2}=\boldsymbol{p}_{i j}^{2}-\left(p_{i}^{0}-p_{j}^{0}\right)^{2}+\gamma_{i j}^{2}\left(\frac{m_{i}^{2}-m_{j}^{2}}{p_{i}^{0}+p_{j}^{0}}\right)^{2},
\end{aligned}
$$

where the velocity and the $\gamma$ factor between the $i$ th and the $j$ th particle are given by

$$
\boldsymbol{\beta}_{i j}=\frac{\boldsymbol{p}_{i}+\boldsymbol{p}_{j}}{p_{i}^{0}+p_{j}^{0}}, \quad \gamma_{i j}=\frac{1}{\sqrt{1-\boldsymbol{\beta}_{i j}^{2}}} .
$$

We now write the explicit form of the equations of motion in $\mathrm{RQMD} / \mathrm{S}$, which is used in the actual simulation. As explained in Sec. II, we use the following potentials:

$$
\begin{aligned}
V= & \sum_{i}\left(V_{\text {Sky } i}+V_{\text {mom } i}\right) \\
= & \sum_{i}\left[\frac{\alpha}{2 \rho_{0}}\left\langle\rho_{i}\right\rangle+\frac{\beta}{(1+\gamma) \rho_{0}^{\gamma}}\left\langle\rho_{i}\right\rangle^{\gamma}\right. \\
& \left.+\sum_{k=1,2} \frac{C_{\mathrm{ex}}^{(k)}}{2 \rho_{0}} \sum_{j(\neq i)} \frac{1}{1+\left[\tilde{\boldsymbol{p}}_{i j} / \mu_{k}\right]^{2}} \rho_{i j}\right],
\end{aligned}
$$


where $\left\langle\rho_{i}\right\rangle$ is obtained from a convolution of the Gaussian wave packet:

$$
\begin{aligned}
\left\langle\rho_{i}\right\rangle & \equiv \sum_{j(\neq i)} \int d \boldsymbol{r} \rho_{i}(\boldsymbol{r}) \rho_{j}(\boldsymbol{r})=\sum_{j(\neq i)} \rho_{i j} \\
& =\sum_{j(\neq i)} \frac{1}{(4 \pi L)^{\frac{3}{2}}} \exp \left(-\frac{\tilde{r}_{i j}^{2}}{4 L}\right) .
\end{aligned}
$$

The width parameters $L=2.05(\mathrm{MH}), 2.1(\mathrm{MS}), \quad 1.08(\mathrm{H}$ and $S) \mathrm{fm}^{2}$ are taken from Refs. [55,56]. The equations of motion (A14) and (A15) then become

$$
\begin{aligned}
\frac{d \boldsymbol{r}_{i}}{d \tau} & =\frac{\boldsymbol{p}_{i}}{p_{i}^{0}}+\sum_{j(\neq i)} D_{i j} \frac{\partial \tilde{\boldsymbol{r}}_{i j}^{2}}{\partial \boldsymbol{p}_{i}}+\sum_{j(\neq i)} E_{i j} \frac{\partial \tilde{\boldsymbol{p}}_{i j}^{2}}{\partial \boldsymbol{p}_{i}}, \\
\frac{d \boldsymbol{p}_{i}}{d \tau} & =-\sum_{j(\neq i)} D_{i j} \frac{\partial \tilde{\boldsymbol{r}}_{i j}^{2}}{\partial \boldsymbol{r}_{i}},
\end{aligned}
$$

where

$$
\begin{aligned}
D_{i j}= & \left(-\frac{1}{2 L}\right) \rho_{i j}\left[\frac{\alpha}{2 \rho_{0}}\left(\frac{m_{i}}{p_{i}^{0}}+\frac{m_{j}}{p_{j}^{0}}\right)\right. \\
& \left.+\frac{\gamma}{1+\gamma} \frac{\beta}{\rho_{0}^{\gamma}}\left\{\frac{m_{i}}{p_{i}^{0}}\left\langle\rho_{i}\right\rangle^{\gamma-1}+\frac{m_{j}}{p_{j}^{0}}\left\langle\rho_{j}\right\rangle^{\gamma-1}\right\}\right] \\
& +\left(-\frac{1}{4 L}\right) \frac{1}{2 \rho_{0}} \rho_{i j}\left(\frac{m_{i}}{p_{i}^{0}}+\frac{m_{j}}{p_{j}^{0}}\right) \sum_{k=1,2} \frac{C_{\mathrm{ex}}^{(k)}}{1+\left[\tilde{\boldsymbol{p}}_{i j} / \mu_{k}\right]^{2}}, \\
E_{i j}= & \frac{1}{2 \rho_{0}} \rho_{i j}\left(\frac{m_{i}}{p_{i}^{0}}+\frac{m_{j}}{p_{j}^{0}}\right) \sum_{k=1,2}\left(-\frac{1}{\mu_{k}^{2}}\right) \frac{C_{\mathrm{ex}}^{(k)}}{1+\left(\tilde{\boldsymbol{p}}_{i j} / \mu_{k}\right)^{2}} .
\end{aligned}
$$

The result of the differentials are [61]

$$
\begin{aligned}
\frac{\partial \tilde{\boldsymbol{r}}_{i j}^{2}}{\partial \boldsymbol{p}_{i}}= & \frac{2 \gamma_{i j}^{2}}{p_{i}^{0}+p_{j}^{0}}\left(\boldsymbol{r}_{i j} \cdot \boldsymbol{\beta}_{i j}\right)\left\{\boldsymbol{r}_{i j}+\gamma_{i j}^{2}\left(\boldsymbol{r}_{i j} \cdot \boldsymbol{\beta}_{i j}\right)\left(\boldsymbol{\beta}_{i j}-\frac{\boldsymbol{p}_{i}}{p_{i}^{0}}\right)\right\}, \\
\frac{\partial \tilde{\boldsymbol{r}}_{i j}^{2}}{\partial \boldsymbol{r}_{i}}= & 2 \boldsymbol{r}_{i j}+2 \gamma_{i j}^{2}\left(\boldsymbol{r}_{i j} \cdot \boldsymbol{\beta}_{i j}\right) \boldsymbol{\beta}_{i j}, \\
\frac{\partial \tilde{\boldsymbol{p}}_{i j}^{2}}{\partial \boldsymbol{p}_{i}}= & 2 \boldsymbol{p}_{i j}-2\left(p_{i}^{0}-p_{j}^{0}\right) \frac{\boldsymbol{p}_{i}}{p_{i}^{0}} \\
& +2 \gamma_{i j}^{4} \frac{1}{p_{i}^{0}+p_{j}^{0}}\left(\frac{m_{i}^{2}-m_{j}^{2}}{p_{i}^{0}+p_{j}^{0}}\right)^{2}\left(\boldsymbol{\beta}_{i j}-\frac{\boldsymbol{p}_{i}}{p_{i}^{0}}\right) . \quad \text { (A29) }
\end{aligned}
$$

Finally, let us check the nonrelativistic limit to confirm the validity of Eqs. (A12)-(A15). We define the kinetic energy as $\mathcal{E}_{i} \equiv p_{i}^{0}-m_{i} c^{2}$ (here we write the speed of light $c$ explicitly), Indeed Hamiltonian (A12) and the equations of motion have the correct nonrelativistic limit, as

$$
\begin{aligned}
& H \approx \sum_{j=1}^{N} \frac{1}{2} \frac{1}{\mathcal{E}_{j} / c^{2}+m_{j}}\left(\frac{\mathcal{E}_{j}^{2}}{c^{2}}+2 m_{j} \mathcal{E}_{j}-\boldsymbol{p}_{j}^{2}-2 m_{j} V_{j}\right) \\
& \underset{c \rightarrow \infty}{\approx} \sum_{j=1}^{N}\left(\mathcal{E}_{j}-\frac{\boldsymbol{p}_{j}^{2}}{2 m_{j}}-V_{j}\right)=E-H_{\mathrm{N} . \mathrm{R} .}, \\
& \frac{d \boldsymbol{r}_{i}}{d \tau}=\frac{\partial H_{\mathrm{N} . \mathrm{R} .}}{\partial \boldsymbol{p}_{i}} \approx \frac{\boldsymbol{p}_{i}}{m_{i}}+\sum_{j=1}^{N} \frac{\partial V_{j}}{\partial \boldsymbol{p}_{i}}, \\
& \frac{d \boldsymbol{p}_{i}}{d \tau}=-\frac{\partial H_{\mathrm{N} . \mathrm{R} .}}{\partial \boldsymbol{r}_{i}} \approx-\sum_{j=1}^{N} \frac{\partial V_{j}}{\partial \boldsymbol{r}_{i}} .
\end{aligned}
$$

[12] K. G. R. Doss et al., Phys. Rev. Lett. 57, 302 (1986).

[13] N. Bastid et al. (FOPI Collaboration), Nucl. Phys. A622, 573 (1997).

[14] A. Andronic et al. (FOPI Collaboration), Phys. Rev. C 67, 034907 (2003).

[15] G. Stoicea et al. (FOPI Collaboration) and P. Danielewicz, Phys. Rev. Lett. 92, 072303 (2004).

[16] R. Pak, O. Bjarki, S. A. Hannuschke, R. A. Lacey, J. Lauret, W. J. Llope, A. Nadasen, N. T. B. Stone, A. M. Vander Molen, and G. D. Westfall, Phys. Rev. C 54, 2457 (1996).

[17] P. Chung et al. (E895 Collaboration) and P. Danielewicz, Phys. Rev. C 66, 021901(R) (2002).

[18] J. Barrette et al. (E877 Collaboration), Phys. Rev. C 55, 1420 (1997); 56, 3254 (1997).

[19] C. Pinkenburg et al. (E895 Collaboration), Phys. Rev. Lett. 83, 1295 (1999).

[20] H. Liu et al. (E895 Collaboration), Phys. Rev. Lett. 84, 5488 (2000).

[21] J. L. Klay et al. (E895 Collaboration), Phys. Rev. Lett. 88, 102301 (2002). 
[22] H. Appelshäuser et al. (NA49 Collaboration), Phys. Rev. Lett. 80, 4136 (1998).

[23] H. Appelshäuser et al. (NA49 Collaboration), Phys. Rev. Lett. 82, 2471 (1999).

[24] C. Alt et al. (NA49 Collaboration), Phys. Rev. C 68, 034903 (2003).

[25] P. K. Sahu, W. Cassing, U. Mosel, and A. Ohnishi, Nucl. Phys. A672, 376 (2000).

[26] P. Danielewicz, Roy A. Lacey, P.-B. Gossiaux, C. Pinkenburg, P. Chung, J. M. Alexander, and R. L. McGrath, Phys. Rev. Lett. 81, 2438 (1998).

[27] P. Danielewicz, Nucl. Phys. A673, 375 (2000).

[28] P. Danielewicz, R. Lacey, and W. G. Lynch, Science 298, 1592 (2002).

[29] R. J. M. Snellings, H. Sorge, S. A. Voloshin, F. Q. Wang, and N. Xu, Phys. Rev. Lett. 84, 2803 (2000).

[30] D. Persram and C. Gale, Phys. Rev. C 65, 064611 (2002).

[31] A. B. Larionov, W. Cassing, C. Greiner, and U. Mosel, Phys. Rev. C 62, 064611 (2000).

[32] T. Maruyama, W. Cassing, U. Mosel, S. Teis, and K. Weber, Nucl. Phys. A573, 653 (1994).

[33] P. K. Sahu and W. Cassing, Nucl. Phys. A712, 357 (2002).

[34] H. Stöcker, E. L. Bratkovskaya, M. Bleicher, S. Soff, and X. Zhu, J. Phys. G 31, S929 (2005).

[35] See, for example, K. Redlich, F. Karsch, and A. Tawfik, J. Phys. G 30, S1271 (2004) [arXiv:nucl-th/0404009]; F. Karsch, Prog. Theor. Phys. Suppl. 153, 106 (2004) [arXiv:hep-lat/0401031]; C. R. Allton, M. Döring, S. Ejiri, S. J. Hands, O. Kaczmarek, F. Karsch, E. Laermann, and K. Redlich, Phys. Rev. D 71, 054508 (2005) [arXiv:hep-lat/0501030], and references therein.

[36] See, for example, Nucl. Phys. A698 (2002), and references therein.

[37] C. Adler et al. (STAR Collaboration), Phys. Rev. C 66, 034904 (2002); B. B. Back et al. (PHOBOS Collaboration), Phys. Rev. Lett. 89, 222301 (2002); S. S. Adler et al. (PHENIX Collaboration), ibid. 91, 182301 (2003); J. Adams et al. (STAR Collaboration), ibid. 92, 062301 (2004).

[38] S. Hama, B. C. Clark, E. D. Cooper, H. S. Sherif, and R. L. Mercer, Phys. Rev. C 41, 2737 (1990).
[39] H. Sorge, H. Stöcker, and W. Greiner, Ann. Phys. 192, 266 (1989).

[40] H. Sorge, Phys. Rev. C 52, 3291 (1995).

[41] H. Sorge, Phys. Lett. B402, 251 (1997); Phys. Rev. Lett. 82, 2048 (1999).

[42] T. Maruyama et al., Nucl. Phys. A534, 720 (1991).

[43] T. Maruyama et al., Prog. Theor. Phys. 96, 263 (1996).

[44] Y. Pang, T. J. Schlagel, and S. H. Kahana, Nucl. Phys. A544, 435c (1992).

[45] B. A. Li and C. M. Ko, Phys. Rev. C 52, 2037 (1995).

[46] W. Ehehalt and W. Cassing, Nucl. Phys. A602, 449 (1996).

[47] L. A. Winckelmann et al., Nucl. Phys. A610, 116c (1996).

[48] S. A. Bass et al., Prog. Part. Nucl. Phys. 41, 255 (1998).

[49] Y. Nara, N. Otuka, A. Ohnishi, K. Niita, and S. Chiba, Phys. Rev. C 61, 024901 (2000).

[50] P. F. Kolb, P. Huovinen, U. W. Heinz, and H. Heiselberg, Phys. Lett. B500, 232 (2001); P. Huovinen, P. F. Kolb, U. W. Heinz, P. V. Ruuskanen, and S. A. Voloshin, ibid. B503, 58 (2001); P. F. Kolb, U. W. Heinz, P. Huovinen, K. J. Eskola, and K. Tuominen, Nucl. Phys. A696, 197 (2001); P. Huovinen, arXiv:nucl-th/0505036; T. Hirano, Phys. Rev. C 65, 011901(R) (2001).

[51] T. Hirano and M. Gyulassy, arXiv:nucl-th/0506049.

[52] X.-N. Wang and M. Gyulassy, Phys. Rev. D 44, 3501 (1991).

[53] T. Sjöstrand et al., Comput. Phys. Commun. 135, 238 (2001).

[54] J. Aichelin, A. Rosenhauer, G. Peilert, H. Stoecker, and W. Greiner, Phys. Rev. Lett. 58, 1926 (1987).

[55] T. Maruyama, K. Niita, K. Oyamatsu, T. Maruyama, S. Chiba, and A. Iwamoto, Phys. Rev. C 57, 655 (1998).

[56] J. Aichelin and H. Stöcker, Phys. Lett. B176, 14 (1986).

[57] J.-Y. Ollitrault, Nucl. Phys. A638, 195c (1998).

[58] S. Soff, S. A. Bass, M. Bleicher, H. Stöcker, and W. Greiner, arXiv:nucl-th/9903061.

[59] A. Komar, Phys. Rev. D 18, 1881 (1978); 18, 1887 (1978); 18, 3617 (1978).

[60] P. A. M. Dirac, Rev. Mod. Phys. 21, 392 (1949).

[61] Y. Hirata et al., Nucl. Phys. A707, 193 (2002); Y. Hirata, Ph.D. thesis, Hokkaido University (2000). 